\title{
Simultaneous Determination of Dopamine and L-Ascorbic Acid by Modified Carbon Paste Electrode with Ni (II) Cyclam Complex
}

\author{
Leila Farzin $^{1}$, Mohammad Reza Milani Hosseini ${ }^{2}$ \\ ${ }^{1}$ Environmental Laboratory, Nuclear Science Research School, Nuclear Science \& Technology Research Institute, \\ Atomic Energy Organization of Iran (AEOI), Tehran, Iran \\ ${ }^{2}$ Analytical Department, Chemistry faculty, Iran University of Science and Technology, Tehran, Iran \\ E-mail:LFarzin84@yahoo.com \\ Received November 8, 2010; revised January 10, 2011; accepted May 16, 2011
}

\begin{abstract}
The electroanalysis of dopamine (DA) and ascorbic acid (AA) by square wave voltammetry has been performed at a modified carbon paste electrode with macrocyclic ligand 1,4,8,11-tetraazacyclotetradecane (cyclam) and monolayer of Ni (II) cyclam. In pH 7.2 buffer solutions, the electrostatic reaction of AA with di-positive monolayer shifts the oxidation potential to less positive potential, while the electrostatic repulsion of DA with the monolayer shifts the oxidation potential of DA to more positive potential. The separation between the oxidation peaks of AA and DA at the present di-positive monolayer modified electrode $(252 \mathrm{mV})$ was larger than that $(187 \mathrm{mV})$ at the cyclam modified electrode. In addition, the catalytic oxidation of AA by oxidized DA has been advantageously eliminated at the modified carbon paste electrode with cyclam and Ni (II) cyclam complex. Thus, the determination of DA in the presence of an excess of AA is possible with the present modified electrodes.
\end{abstract}

Keywords: Macrocyclic Compound, Carbon Paste Electrode, Dopamine, Ascorbic Acid

\section{Introduction}

Dopamine (DA) and ascorbic acid (AA) are compounds of great biomedical and neurochemical interest playing a potential role in human metabolism. DA is one of the most significant catecholamine, functioning as a neurotransmitter in the central nervous system and a medicament to drug addiction and Parkinson's disease [1,2]. It affects brain processes that control movement, emotional response, and ability to experience pleasure and pain. AA is a water-soluble vitamin that is widely required in metabolism. It has been used in the prevention and treatment of common cold, mental illness, cancer and Aids [3]. In mammalian brain DA and AA coexists in the extracellular fluids.

There are various determination methods includes ultraviolet spectroscopy (UV) [4], high performance liquid chromatography (HPLC) [5,6], capillary electrophoresis (CE) [7] and electrochemical approaches [8-10]. Because both DA and AA are oxidisable compounds, their detection can be made by electrochemical methods based on anodic oxidation. When a potential is applied at the electrode, ascorbic acid is also oxidized to dehydroascorbic acid, which undergoes further chemical reaction to form the gem-diol (Figure 1). As is known the oxidation potential is $\mathrm{pH}$ dependent [11] (The first $\mathrm{pK}_{\mathrm{a}}$ is at 4.17 and the second is at 11.57).

Dopamine is oxidized to form dopaminequinone with the liberation of two electrons (Figure 2). It is generally believed that direct redox reactions of these species at bare electrodes are irreversible and therefore require high overpotentials. Moreover the direct redox reactions of these species at the bare electrodes take place at very similar potentials and often suffer from a pronounced
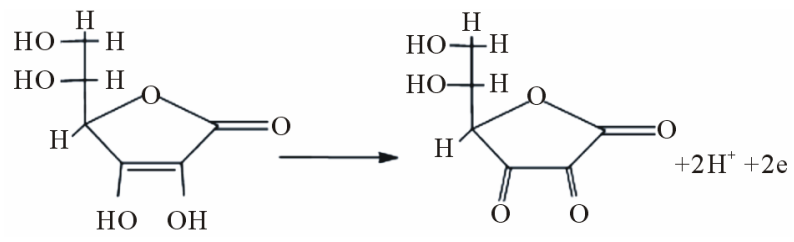

Figure 1. Electrooxidation of ascorbic acid. 


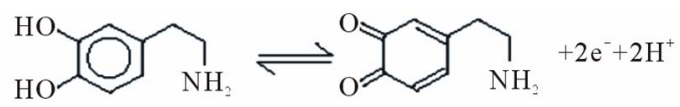

Figure 2. Electrooxidation of dopamine.

fouling effect, which results in rather poor selectively and reproducibility. Thus the simultaneous determination of DA and AA is of critical importance in the field of biochemistry and medical treatment [12].

The use of chemically modified electrodes greatly increases the selectivity and sensitivity toward these analytes. The development of voltammetric sensors for the detection of neurotransmitters in the extracellular fluid of the central nervous system has received much interest in the past few decades. So, many different strategies have been employed for the modification of the electrode surface [13]. A simple method for preparing electrochemical modified electrodes (CME) is based on doping carbon paste with the biocatalyst [14-16]. The most important advantage of the "mixed catalyst-carbon paste electrode" is the substantial reduction of response time owing to the absence of a layer that hinders mass transport. The catalyst is an integral part of the sensing element, and hence the electrode responds rapidly to changes in the level of the substrate.

The goal of this study was to develop a production method for modified carbon paste electrodes with macrocyclic compounds for selective measurement of neurotransmitters in physiological $\mathrm{pH}$ (7.2). This modified electrode with $\mathrm{Ni}$ (II) 1, 4, 8, 11 -tetraazacyclotetradecane has been used for Simultaneous Determination of DA and AA. The electrochemical behavior of a broad family of macrocyclic complexes of nickel has been studied by Busch et al. [17].

\section{Materials and Methods}

\subsection{Chemical and Reagents}

All chemicals used were of analytical-reagent grade. Double distilled, deionized water (Milli-Q system, Millipore, Japan) was used for preparation of all solutions. 1,4,8,11-tetraazacyclotetradecane (cyclam), dopamine hydrochloride and ascorbic acid were bought from Fluka and used as such. All the voltammetric studies were carried out in phosphate buffer (potassium phosphate were also used as a supporting electrolyte). Buffer solutions were prepared from orthophosphoric acid and its salts in the $\mathrm{pH}$ range of 3-9.

\subsection{Apparatus}

The voltammetric system used for the studies was Autolab PSTAT 10 potentiostat joined to a Metrohm 663 VA.
Square wave voltammetry was carried out in a threeelectrode cell. Silver/silver chloride $\left(3 \mathrm{~mol} \cdot \mathrm{dm}^{-3} \mathrm{KCl}\right)$, a platinum wire and a bare or modified electrode were used as reference, counter and working electrodes, respectively. The $\mathrm{pH}$ values were measured with a digital $\mathrm{pH}$ meter MK VI (systronics).

\subsection{Preparation of Bare Carbon Paste Electrode}

The bare carbon paste electrode was prepared by hand mixing of graphite powder and silicon oil at a ratio 70:30 $(\mathrm{w} / \mathrm{w})$ in an agate mortar until a homogenous paste was obtained. The paste was then tightly packed into a PVC tube (3 $\mathrm{mm}$ internal diameter) and the electrical contact was provided by a copper wire connected to the end of tube. The bare carbon paste electrode was polished successively with 0.3 and $0.05 \mu \mathrm{m} \mathrm{Al}_{2} \mathrm{O}_{3}$ slurry on emery paper. It was then rinsed with doubly distilled water and sonicated in $1+1 \mathrm{HNO}_{3}$, acetone and doubly distilled water for $10 \mathrm{~min}$, respectively.

\subsection{Preparation 1of Modified Carbon Paste Electrode}

The macrocyclic nickel complex (nickel (II) 1,4,8,11tetraazacyclotetradecane) was synthesized, purified and characterized by elemental analysis and ${ }^{13} \mathrm{C}-\mathrm{NMR}$ and IR spectral measurements according to reported procedure [18].

The carbon paste electrodes were prepared as before with $5 \%$ of the modifier in graphite-silicon oil matrix and used in conjunction with an $\mathrm{Ag} / \mathrm{AgCl}$ reference electrode and a platinum counter electrode. The thickness of modified carbon paste was controlled in the range of $4-6$ $\mathrm{mm}$.

\subsection{Procedure}

The electrochemical experiments were performed in a 25 $\mathrm{cm}^{3}$ electrolytic cell with $10 \mathrm{~cm}^{3}$ solutions. All measurements were conducted at room temperature and under a nitrogen atmosphere. Nitrogen gas was bubbled through the solution for $30 \mathrm{~min}$ prior to each electrochemical measurement.

Solutions of various $\mathrm{pHs}$ were tested with phosphate buffer. The square-wave voltammograms were recorded for the unmodified electrode and the electrode modified with cyclam (CME-1) in $0.5 \mathrm{M}$ phosphate buffer solution $(\mathrm{pH}=7.2)$ containing $\mathrm{DA}$ and AA at different scan rates. Then, another set of experiments was carried out to study the effect of catalysis by the incorporated metal ion in the macrocyclic ring on the electrode modified with nickel (II) macrocyclic complex (CME-2). 


\section{Results and Discussion}

\subsection{Electrochemical Behavior of AA and DA on CME-1}

The square-wave voltammograms obtained for the oxidation of AA and DA at the CME-1 and the bare electrode are shown in Figure 3. At the bare electrode when both AA and DA coexist, only one voltammetric peak is obtained for both analytes. Thus it is impossible to determine the individual concentrations from the broad voltammetric peak. Moreover the catalytic oxidation of AA by the oxidized DA [19] enhances the oxidation peak current of DA at the bare electrode. The precise determination of DA in the presence of AA is not possible because of this catalytic oxidation. As the AA concentration, for example, in the extracellular fluid is very high, this mediated oxidation would affect the accurate determination of DA and this unwanted catalytic oxidation needs to avoid. The present modified electrode clearly separates the merged voltammetric peaks of AA and DA and the mediated oxidation of AA by oxidized DA has been successfully eliminated. At these electrodes the AA oxidation occurs well before the DA oxidation potential and hence the mediated oxidation would not be expected. Since the voltammetric peak of DA is well separated from the AA peak, the determination of DA in the presence of AA is possible with the present modified electrode. The separation between the oxidation peaks of AA and DA at the CME-1 in phosphate buffer solution $(\mathrm{pH}=$ 7.2) was $187 \mathrm{mV}$.

\subsection{Electrochemical Behavior of AA and DA on CME-2}

Figure 4 shows the oxidation of AA and DA at the bare

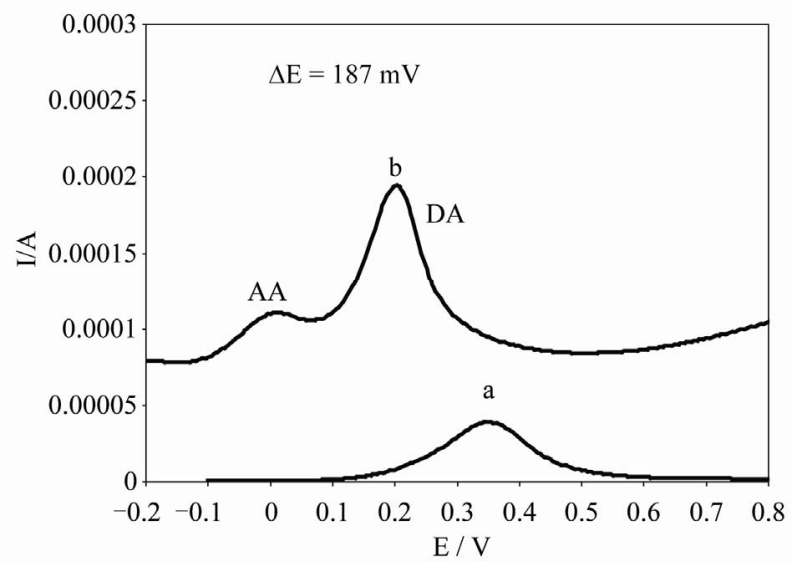

Figure 3. Square-wave voltammograms obtained for the oxidation of DA and AA $\left(2 \times 10^{-5} \mathrm{M}\right)$ at (a) bare and (b) CME-1 in $0.5 \mathrm{M}$ phosphate buffer $(\mathrm{pH}=7.2)$.

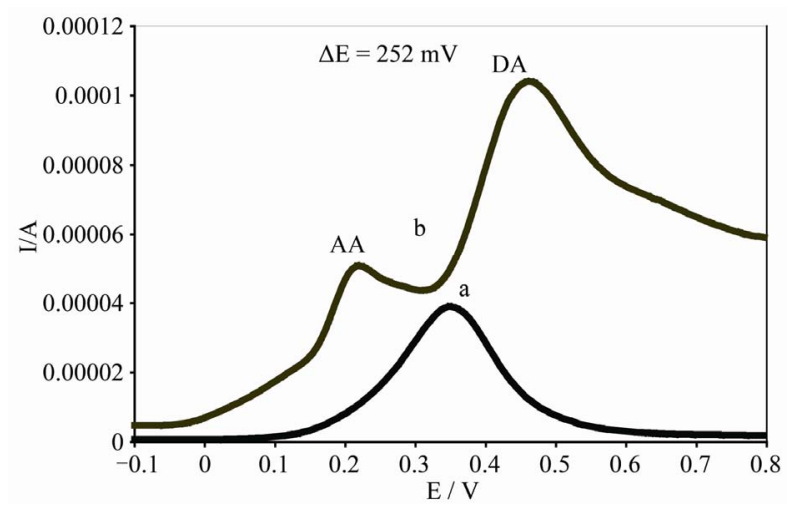

Figure 4. Square-wave voltammograms obtained for the oxidation of DA and $\mathrm{AA}\left(2 \times 10^{-5} \mathrm{M}\right)$ at (a) bare and (b) CME-2 in $0.5 \mathrm{M}$ phosphate buffer $(\mathrm{pH}=7.2)$.

carbon paste electrode and the modified carbon paste electrode with nickel (II) macrocyclic complex (CME-2). As can be readily seen from Figure $\mathbf{4}$ a negative shift in the AA oxidation potential can be the electrostatic interaction of AA with the positively charged monolayer. Since AA is negatively charged in neutral aqueous solution $(\mathrm{pH}=7.2)$, the electrostatic interaction is expected between $\mathrm{AA}$ and $\mathrm{Ni}^{2+}$ redox centers of the monolayer and it would favor the oxidation of AA.

In contrast, DA exists in the cationic form physiological $\mathrm{pH}\left(\mathrm{pK}_{\mathrm{a}} 8.9\right)$. It is repelled by the $\mathrm{Ni}^{2+}$ redox centers. Hence, it cannot enter the monolayer to the same extent as $\mathrm{AA}$, and the interference with the determination of DA is diminished. These results indicated that the problem of the overlapped voltammetric responses of DA with AA, due to their coexistence in real biological fluids can be effectively overcome by use of dipositive monolayer of $\mathrm{Ni}$ (II) cyclam.

The monolayer modified electrode successfully resolves the merged voltammetric peaks of AA and DA and the peaks are separated enough (252 $\mathrm{mV}$ separation) to determine the concentration of each analytic.

\subsection{Effect of Film Thickness on the Voltammetric Response}

The thickness of complex film directly controls the electrode performance. The optimum film thickness reflects compromise between mechanical stability and residual current.

Nevertheless, the high residual current remains a severe limitation of these modified electrodes. The residual current tends to be high when maximum catalyst is used for modification of carbon paste electrode. The film thickness was varied by preparing the electrodes with different $w t \%$ of catalyst. The electrode prepared with $5 \mathrm{wt} \%$ macrocyclic complex or ligand, shows the best performance. When the films were too thin, the limited amount of 
catalyst loaded apparently affect the sensitivity. Whereas, when the films were too thick, residual current increased remarkably. So, electrodes prepared with the optimum of catalyst $(5 \mathrm{wt} \%)$, were used in all experiments.

\subsection{Optimization of the Solution pH}

Figure 5 shows the $\Delta \mathrm{E}_{\mathrm{p}}$ versus $\mathrm{pH}$ plots for CME-2 in the phosphate buffer with various $\mathrm{pHs}$ (in range of 3 - 9).

The electrochemical reaction can be induced and monitored by voltammetry to quantify the concentration of ascorbic acid in solution.

The voltammograms obtained with the Ni (II) macrocyclic for solutions containing L-ascorbic acid in strongly acidic media (e.g. pH2) showed that L-ascorbic acid did not couple catalytically with the Ni (II) macrocyclic. Therefore, optimization of the solution $\mathrm{pH}$ was necessary in order to obtain a catalytic couple. A variation in the electrolyte $\mathrm{pH}$ will result in variations in the formal potential of L-ascorbic acid. Therefore, the thermodynamic driving force for the catalysis will vary with the $\mathrm{pH}$, making the peak currents and the shapes of the voltammograms at different $\mathrm{pH}$ values. The anodic peak currents increased with an increase in the $\mathrm{pH}$ up to 6.6, and then gradually decreased up to $\mathrm{pH} 9$. So, the most optimized $\mathrm{pH}$ for catalytic oxidation of AA is 6.6. However, in this $\mathrm{pH}$, electron transfer kinetic for the oxidation of DA was found to be rather sluggish owing to the electrostatic repulsion between positively charged $\mathrm{DA}\left(\mathrm{pK}_{\mathrm{a}} 8.9\right)$ and $\mathrm{Ni}$ (II). So, the electrostatic repulsion of DA and with di-positive monolayer shifts the oxidation potential of DA to more positive potential, while the electrostatic reaction of $\mathrm{AA}$ with the monolayer shifts the oxidation potential to less positive potential. In this study, all of the measurements were carried out at the physiological $\mathrm{pH}$ (7.2) that it is much near to optimized $\mathrm{pH}$.

\subsection{Effect of Scan Rate on Peak Currents of DA and $A A$}

The results show an initial linearity which curves off at

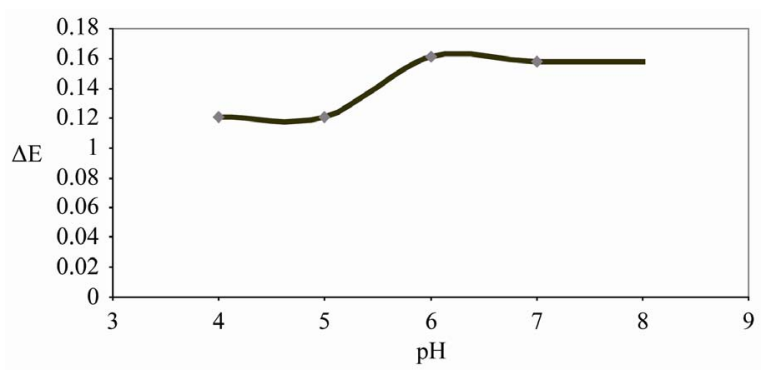

Figure 5. Plot of $\Delta E_{p}$ vs $p H$ for $2 \times 10^{-3} M$ ascorbic acid and dopamine obtained by CME-2 in the $0.5 \mathrm{M}$ phosphate buffer. higher scan rates. It suggests that the reaction is initially diffusion controlled, but at faster scan rates the electron transfer becomes rate determining. It appears from these data that a scan rate of $100 \mathrm{mV} \cdot \mathrm{s}^{-1}$ was used for the purpose of simultaneous determination of DA and AA. In addition, the $E_{p}$ values are shifted to more positive values.

\subsection{Calibration Curve and Reproducibility}

The calibration plots for the oxidation of AA and DA were linear for a wide range of concentration (1-100 $\mu \mathrm{M}$ for AA and $1.5-100 \mu \mathrm{M}$ for DA at the CME-2).

To characterize the reproducibility of the CME-2, repetitive measurementregeneration cycles were carried out. The results of 15 successive measurements showed a relative standard deviation of $4.1 \%$ and $3.8 \%$ for $50 \mu \mathrm{M}$ ascorbic acid and $50 \mu \mathrm{M}$ dopamine.

\section{Conclusions}

Redox processes of organic compounds often have slow charge transfer rates, leading to poorly defined voltammetric responses. Modification of the electrode surface by a redox mediator reduces the over-potential for the redox processes. The modified carbon paste electrodes with cyclam and $\mathrm{Ni}$ (II) cyclam monolayer have been successfully applied to the determination of ascorbic acid and dopamine. The separation between the oxidation peaks of AA and DA at the CME-2 $(252 \mathrm{mV})$ was larger than that $(187 \mathrm{mV})$ at the CME-1.

The carbon paste approach permits convenient mixing of different ligands and as the ligand is homogeneously mixed in the bulk of the paste, renewal of the surface is done simply by pressing out the paste from syringe, which is easier and faster. Moreover, the electrode so fabricated can be stored for about six months in an airtight container.

\section{Reference}

[1] H. Zhao, Y. Zhang and Z. Yuan, "Electrochemical Determination of Dopamine Using a Poly (2-Picolinic Acid) Modified Glassy Carbon Electrode," Analyst, Vol. 126, No. 3, 2001, pp. 358-360. doi:10.1039/b008283j

[2] T. E. Smith, "Text Book of Biochemistry with Clinical Correlations," T. M. Devlin Eds., Wiley/Liss, New York, 1992, p. 929.

[3] O. Gilbert, U. Chandra, B. E. Kumara Swamy, M. Panduranga Char, C. Nagaraj and B. S. Sherigara, "Poly (Alanine) Modified Carbon Paste Electrode for Simultaneous Detection of Dopamine and Ascorbic Acid," International Journal of Electrochemical Science, Vol. 3, No. 3, 2008, pp. 1186-1195. 
[4] M. Rohani Moghadam, S. Dadfarnia, A. M. Haji Shabani and P. Shahbazikhah, "Chemometric-Assisted KineticSpectrophotometric Method for Simultaneous Determination of Ascorbic Acid, Uric Acid, and Dopamine," Analytical Biochemistry, Vol. 410, No. 2, 2011, pp. 289-295. doi:10.1016/j.ab.2010.11.007

[5] W. Zhang, X. Cao, Y. Xie, S. Ai, L. Jin and J. Jin, "Simultaneous Determination of the Monoamine Neurotransmitters and Glucose in Rat Brain by Microdialysis Sampling Coupled with Liquid Chromatography-Dual Electrochemical Detector," Journal of Chromatography B, Analytical Technologies in the Biomedical and Life Sciences, Vol. 785, No. 2, 2003, pp. 327-336. doi:10.1016/S1570-0232(02)00953-4

[6] C. Muzzi, E. Bertocci, L. Terzuoli, B. Porcelli, I. Ciari, R. Pagani and R. Guerranti, "Simultaneous Determination of Serum Concentrations of Levodopa, Dopamine, 3-OMethyldopa and $\alpha$-Methyldopa by HPLC," Biomedicine \& Pharmacotherapy, Vol. 62, No. 4, 2008, pp. 253-258. doi:10.1016/j.biopha.2007.10.018

[7] H. Li, C. Li, Z. Y. Yan, J. Yang and H. Chen, "Simultaneous Monitoring Multiple Neurotransmitters and Neuromodulators During Cerebral Ischemia/Reperfusion in Rats by Microdialysis and Capillary Electrophoresis," Journal of Neuroscience Methods, Vol. 189, No. 2, 2010, pp. 162-168. doi:10.1016/j.jneumeth.2010.03.022

[8] M. Pandurangachar, B. E. Kumara Swamy, B. N. Chandrashekar, O. Gilbert and B. S. Sherigara "Electrochemical Deposition of 1-Butyl-4-Methyl-Pyridinium Tetrafluroborate Ionic Liquid on Carbon Paste Electrode and its Application for the Simultaneous Determination of Dopamine, Ascorbic Acid and Uric Acid," Journal of Molecular Liquids, Vol. 158, No. 1, 2011, pp. 13-17. doi:10.1016/j.molliq.2010.09.005

[9] R. Zhang, G. D. Jin, D. Chen and X. Y. Hu "Simultaneous Electrochemical Determination of Dopamine, Ascorbic Acid and Uric Acid Using Poly (Acid Chrome Blue K) Modified Glassy Carbon Electrode," Sensors and Actuators B: Chemical, Vol. 138, No. 1, 2009, pp. 174-181.

[10] A. A. Ensafi, M. Taei, T. Khayamian and A. Arabzadeh "Highly Selective Determination of Ascorbic Acid, Dopamine, and Uric Acid by Differential Pulse Voltammetry Using Poly (Sulfonazo III) Modified Glassy Carbon Electrode," Sensors and Actuators B: Chemical, Vol. 147, No. 1, 2010, pp. 213-221. doi:10.1016/j.snb.2010.02.048

[11] V. S. Lieri, M. Algarra and A. Martins, "Electrocatalytic Determination of Vitamin C Using Calixarene Modified
Carbon Paste Electrodes," Electroanalysis, Vol. 16, No. 24, 2004, pp. 2082-2086. doi:10.1002/elan.200403054

[12] Z. Gao and H. Huang, "Simultaneous Determination of Dopamine, Uric Acid and Ascorbic Acid at an Ultrathin Film Modified Gold Electrode," Chemical Communications, Vol. 8, No. 1, 1998, pp. 2107-2108. doi: $10.1039 / \mathrm{a} 805915 \mathrm{~b}$

[13] C. R. Raj and T. Ohsaka, "Electroanalysis of Ascorbate and Dopamine at a Gold Electrode Modified with a Positively Charged Self-Assembled Monolayer," Journal of Electroanalytical Chemistry, Vol. 496, No. 1-2, 2001, pp. 44-49. doi:10.1016/S0022-0728(00)00335-1

[14] J. Tashkhourian, M. R. Hormozi Nezhad, J. Khodavesi and S. Javadi, "Silver Nanoparticles Modified Carbon Nanotube Paste Electrode for Simultaneous Determination of Dopamine and Ascorbic Acid," Journal of Electroanalytical Chemistry, Vol. 633, No. 1, 2009, pp. 85-91. doi:10.1016/j.jelechem.2009.04.028

[15] O. Gilbert, B. E. Kumara Swamy, U. Chandra and B. S. Sherigara, "Simultaneous Detection of Dopamine and Ascorbic Acid Using Polyglycine Modified Carbon Paste Electrode: A Cyclic Voltammetric Study," Journal of Electroanalytical Chemistry, Vol. 636, No. 1-2, 2009, pp. 80-85.

[16] B. N. Chandrashekar, B. E. Kumara Swamy, M. Pandurangachar, S. S. Shankar, O. Gilbert, J. G. Manjunatha and B. S. Sherigara, "Electrochemical Oxidation of Dopamine at Polyethylene Glycol Modified Carbon Paste Electrode: A Cyclic Voltammetric Study," International Journal of Electrochemical Science, Vol. 5, No. 5, 2010, pp. 578-592.

[17] M. Khorasani-Motlagh and M. Noroozifar, "Electrocatalytic Determination of L-Ascorbic Acid by Modified Glassy Carbon with $\mathrm{Ni}\left(\mathrm{Me}_{2}\left(\mathrm{CH}_{3} \mathrm{CO}\right)_{2}[14]\right.$ tetraenoN $\left.\mathrm{N}_{4}\right)$ Complex," Analytical Scientific, Vol. 19, No. 12, 2003, pp. 1671-1674. doi:10.2116/analsci.19.1671

[18] Y. Kashiwagi, C. Kikuchi and J. Anzai, "Electrocatalytic Dehalogenation of Organohalides on a $\mathrm{Ni}$ (II) Tetraazamacrocyclic Complex-Modified Graphite Felt Electrode," Journal of Electroanalytical Chemistry, Vol. 518, No. 1, 2002, pp. 51-55. doi:10.1016/S0022-0728(01)00697-0

[19] M. A. Dayton, A. G. Ewing and R. M. Wightman, "Response of Microvoltammetric Electrodes to Homogeneous Catalytic and Slow Heterogeneous Charge-Transfer Reactions," Analytical Chemistry, Vol. 52, No. 14, 1980, pp. 2392-2396. doi:10.1021/ac50064a035 